\title{
Variations in the Circumflex Branches of the Profunda Femoris Artery - A Cadaveric Study
}

\author{
Anne George ${ }^{1}$, Maheswary Thampi Santhakumary² \\ ${ }^{1,2}$ Department of Anatomy, Govt. Medical College Kottayam, Kerala, India.
}

\section{ABSTRACT}

\section{BACKGROUND}

The external iliac artery passes behind the inguinal ligament into the front of the thigh as the femoral artery (FA). The FA gives off many branches both superficial and deep. The profunda femoris artery (PFA) is one of the deep branches given off in the femoral triangle in front of the thigh. The PFA gives off the medial circumflex femoral artery (MCFA) and the lateral circumflex femoral arteries (LCFA) and continues downwards giving off the first, second and third perforating arteries. The PFA terminates as the fourth perforating artery. Many variations in the circumflex branches of the PFA have been found by various authors. These variations are of great significance during procedures done in front part of the thigh.

\section{METHODS}

This is a descriptive cadaveric study. We dissected the thighs of 57 embalmed bodies. We looked for the medial (MCFA) and lateral (LCFA) circumflex arteries which are branches of PFA. Each artery was followed till its termination. The distance of their origin from the point of the origin of PFA from the FA was measured and noted. The distance between pubic symphysis and anterior superior iliac spine was measured using black silk and measuring scale. The midpoint was marked using skin marking pen and an incision extending from anterior superior iliac spine to pubic symphysis was made. Another incision was made from the above midpoint to the midpoint of a horizontal incision at the level of knee joint. Femoral sheath was identified and incised. Femoral artery, profunda femoris artery and its circumflex branches were identified. The modes of origin of MCFA and LCFA were noted. The distance of origin of these from the origin of PFA were measured. Variations in the branching pattern of MCFA and LCFA were looked for and noted down.

\section{RESULTS}

We found that in $83 \%$ of the total cases MCFA took origin from PFA and its origin was from the FA in $13 \%$. In $84 \%$ of total cases LCFA arose from PFA on the right side and $70 \%$ on the left side. A common stump of origin was noted in 3 cases.

\section{CONCLUSIONS}

Medial and lateral circumflex branches of PFA exhibit wide variations.

\section{KEY WORDS}

Medial Circumflex Femoral Artery, Lateral Circumflex Femoral Artery, Variations in Origin and Branching
Corresponding Author:

Dr. Maheswary Thampi Santhakumary, Anatomy, Govt. Medical College, Kottayam, Kerala, India.,

E-mail: drsmthampi@gmail.com

DOI: $10.14260 /$ jemds/2021/218

How to Cite This Article:

George A, Santhakumary MT. Variations in the circumflex branches of the profunda femoris artery - a cadaveric study. J Evolution Med Dent Sci 2021;10(14):10201024, DOI: 10.14260/jemds/2021/218

Submission 17-06-2019,

Peer Review 24-01-2021,

Acceptance 02-02-2021,

Published 05-04-2021.

Copyright (C) 2021 Anne George et al. This is an open access article distributed under Creative Commons Attribution License [Attribution 4.0 International (CC BY 4.0)] 


\section{BACKGROUND}

Femoral artery (FA) also known as common femoral artery is the chief deep artery of the thigh which begins behind the inguinal ligament at the mid-inguinal point as the continuation of the external iliac artery. In the front of the thigh in the femoral triangle bounded laterally by sartorius and medially by adductor longus and above by the inguinal ligament, the FA gives off three superficial and three deep branches. The superficial branches are the superficial external pudendal, superficial epigastric and superficial circumflex iliac arteries. The deep branches are profunda femoris artery (PFA), deep external pudendal and ascending genicular arteries. The largest branch of the FA is the PFA otherwise known as deep femoral artery. The femoral artery below the origin of PFA is also known as superficial femoral artery. PFA arises from the lateral side of the FA $3-4 \mathrm{cms}$ below the inguinal ligament. ${ }^{1}$ The PFA gives off medial circumflex femoral (MCFA), lateral circumflex femoral (LCFA), three perforating and muscular arteries and itself continues as the fourth perforating artery. ${ }^{2}$

The MCFA arises from the posterolateral aspect of the PFA in the femoral triangle and descends between psoas major and pectineus muscles, passes posteriorly between obturator externus and adductor brevis and enters the gluteal region between quadratus femoris and the upper border of adductor magnus. Here it gives off the ascending, acetabular and transverse branches. It forms the trochanteric anastomosis with the first perforator, inferior gluteal and transverse branch of LCFA. Ascending branches of LCFA anastomose with MCFA forming a vascular ring. Retinacular branches from the vascular ring provide blood to the head and neck of the femur. MCFA is the chief arterial source to the head of femur. The LCFA leaves the femoral triangle by passing deep to the sartorius muscle. It divides into an ascending branch which passes along the intertrochanteric line lateral to the hip joint and anastomoses with superior gluteal artery and the deep circumflex iliac artery at the spinous anastomosis.3,4

The first three perforating arteries piercing the adductor magnus and the lateral intermuscular septum close to the femur, enter the flexor or the posterior compartment of the thigh. They communicate with each other by ascending and descending branches close to the insertion of adductor magnus. The ascending branch of the first perforating artery participates in the formation of cruciate anastomosis. The descending branch of the fourth perforator communicates with the popliteal artery. In this way a potential arterial chain is formed in the back of the thigh.

Branches of LCFA and MCFA anastomose with branches of superior and inferior gluteal arteries. Descending branch of inferior gluteal artery, first perforating artery and transverse branches of MCFA and LCFA form the cruciate anastomosis. Cruciate anastomosis is seen at the back of the thigh in the interval between quadratus femoris and the upper border of adductor magnus. Trochanteric anastomosis is seen on the greater trochanter of the femur. It is formed by the descending branch of the superior gluteal artery and ascending branches of MCFA and LCFA. Obstruction in the FA proximal to the origin of PFA leads to the establishment of the collateral circulation through the anastomosis between PFA and internal and external iliac arteries through the cruciate, trochanteric, spinous anastomoses, around the origin of obturator externus and in the perineum.
Many variations of MCFA and LCFA have been noted in various studies like origin of MCFA and LCFA from femoral artery and common stem of PFA and MCFA. Low and high origin of the artery from PFA should be kept in mind during procedures in the femoral triangle. Proximity of femoral nerve to LCFA should be kept in mind during femoral nerve block. In reconstructive surgery of hip, fixation procedures on the upper end of femur and acetabulum, anatomical knowledge of variation of MCFA is very essential as injury to the artery can lead to avascular necrosis.

LCFA arises from the root of PFA leaves the femoral triangle passing deep to sartorius and to rectus femoris. At this point the internal \& external iliac arteries communicate with branches of PFA forming an anastomotic ring on the femoral neck with the ascending branch of MCFA. Descending branches passes along anterior boarder of vastus lateralis supplying it. One long branch anastomosis with the superior lateral genicular branch of popliteal artery. Transverse branch passes laterally anterior to vastus intermedius then pierces vastus lateralis to enter the posterior compartment to participate in the cruciate anastomosis.

LCFA takes part in spinous, trochanteric, cruciate and genicular anastomoses. ${ }^{5}$ Knowledge of the variations is important in hip joint replacement. 6 Branches of LCFA are used for various bypass surgeries. ${ }^{7-9}$ It is reported that precise knowledge of the anatomy of MCFA is essential in performing both trochanteric and inter trochanteric osteotomies and is also helpful to avoid iatrogenic avascular necrosis of the head of the femur in reconstructive surgery of the hip fixation of acetabular fractures through the posterior approach. ${ }^{10}$ Both circumflex arteries arise independently from the femoral arteries, the PFA in such cases usually having a low origin, and one of the perforating arteries may arise from the circumflex according to a report. ${ }^{11}$ Most commonly PFA originated from the posterolateral surface of the common femoral artery and in two cases (10\%) a common trunk was found for PFA and MCFA. ${ }^{1}$

According to a report ${ }^{12}$ in $78.3 \%$ of cases, the MCFA originated from DFA, in $11.7 \%$ cases from common femoral artery (CFA) and in $5 \%$ cases from superficial femoral artery (SFA). The incidence of MCFA originating from FA was higher than that for LCFA. ${ }^{13}$ According to a report, the ascending branch of LCFA was dominant in the vascularisation of tensor fascia lata muscle flap (TFL). This vessel or its terminal branches enter the TFL muscle at various levels between anterior, superior, iliac crest and its insertion into iliotibial tract, the most common level being midway between the above two points. ${ }^{14}$ It was reported that the lateral circumflex femoral artery arose from the femoral artery in $15 \%$ cases proximal to the PFA. ${ }^{15}$ According to one report. ${ }^{16}$ lateral circumflex femoral artery was used as a perforator flap in the reconstruction of gunshot wounds. In a study where 100 lower extremities (50 left and 50 right) were dissected, MCFA took origin from FA in $15 \%$ of cases. (15 cases). ${ }^{17}$ In a report the descending branches of LCFA could be used as high flow channel for extra cranial-intracranial bypass surgery. In another study, absence of LCFA was seen in 2 lower limbs. Ascending, transverse and descending branches arose from PFA. ${ }^{18}$ In a case presentation, right femoral artery after descending by $4.5 \mathrm{~cm}$ from inguinal ligament gave a common stump which instantly bifurcated into lateral circumflex and profunda femoris arteries. ${ }^{19} \mathrm{~A}$ rare variation of trifurcated 
right femoral artery was reported in a case. The trifurcated arteries from lateral to medial side were lateral circumflex femoral artery, femoral artery and profunda femoris artery. ${ }^{20}$

\section{Objectives}

This study was conducted to find the variations of the circumflex arteries which will definitely help the surgeon to avoid unnecessary iatrogenic complications.

\section{METHODS}

This is a descriptive cadaveric study done between March 2019 to September 2019 after getting permission from institutional review board on $15^{\text {th }}$ March 2019 via IRB number 116 / 2018. Thighs of a total of 57 embalmed bodies were dissected and analysed.

In 57 embalmed bodies, the distance between pubic symphysis and anterior superior iliac spine was measured using black silk and measuring scale. The midpoint was marked using skin marking pen and an incision extending from anterior superior iliac spine to pubic symphysis was made. Another incision was made from the above midpoint to the midpoint of a horizontal incision at the level of knee joint. Femoral sheath was identified and incised. Femoral artery, profunda femoris artery and its circumflex branches were identified. The modes of origin of MCFA and LCFA were noted. The distance of origin of these from the origin of PFA were measured. Variations in the branching pattern of MCFA and LCFA were looked for and noted down. Relevant photographs were taken with a digital camera. Results were analysed and compared with those of other studies. According to a study, it was found that medial circumflex femoral originated from FA including a common stem in 15 cases (15\%). ${ }^{17}$

Since this is a descriptive study, the sample size was calculated using the formula

$\mathrm{N}=\frac{3.84 \mathrm{PQ}}{\mathrm{D}^{2}}$

Where,

$P$ is the incidence in previous study which is $15 \%$ and $Q$ is 100 P i.e. 85 and $\mathrm{D}=10$ (absolute precision).

\section{Statistical Analysis}

Continuous variables were expressed as means and standard deviations and categorical variables were stated as numbers and percentage. The data was tabulated in Excel software sheets. It was statistically analysed using Statistical Package for the Social Sciences (SPSS) version 16.0 for window.

\section{RESULTS}

The present cadaveric study was carried out in the lower limbs of the cadavers in the dissection hall of Govt. Medical College Kottayam. Review of literature showed that there were great variations in the origin of MCFA and LCFA in studies conducted by different authors. Our attempt here was to find the occurrence of variations in MCFA and LCFA in the population in the southern part of India. Previous authors have reported ${ }^{1}$ variations based on race and region.
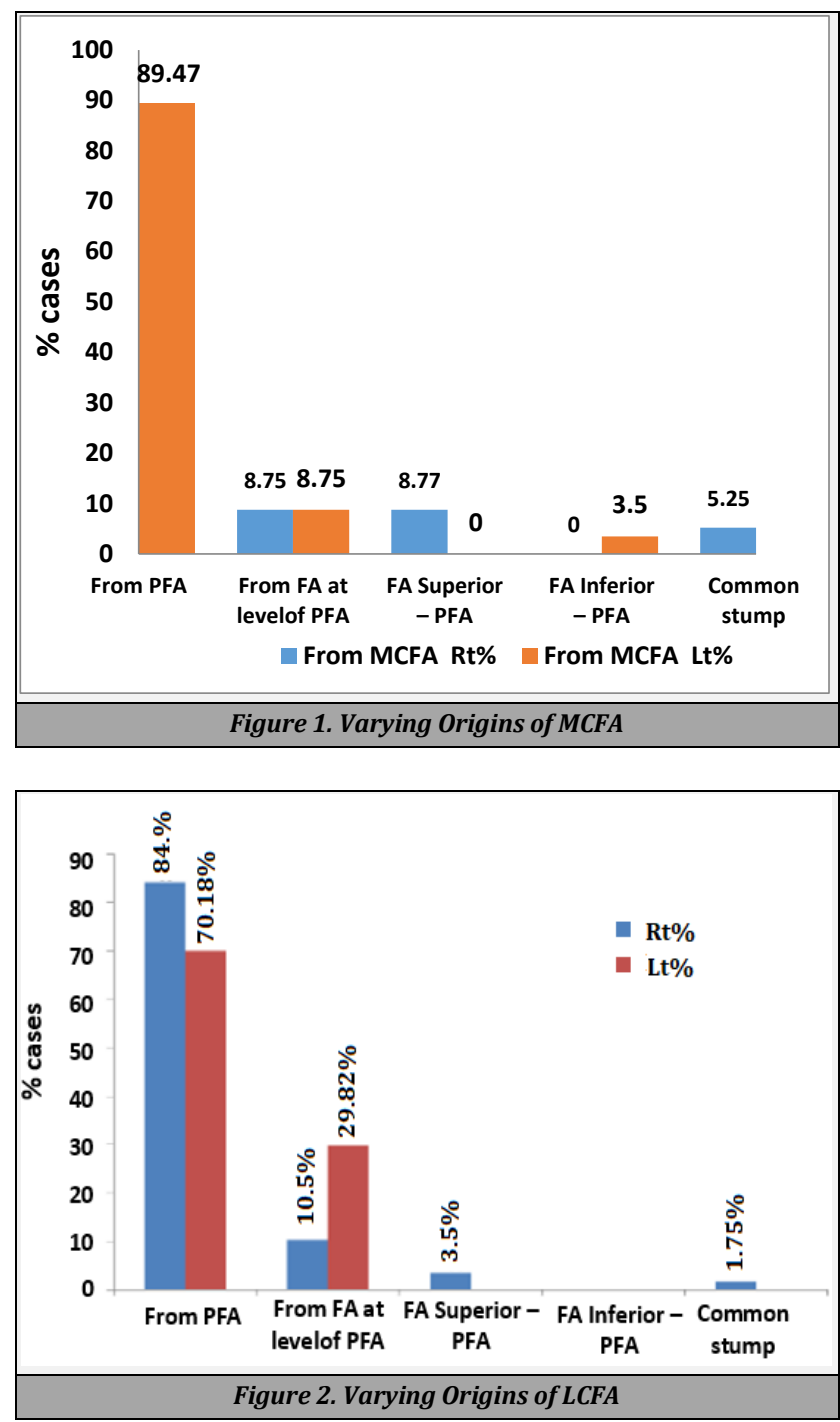

Figure 1 shows varying origins of MCFA. We found that in 44 cases $(77.19 \%)$ on the right side and 51 cases $(89.47 \%)$ on the left side MCFA originated from PFA. This indicated that 83 $\%$ of total cases took origin from PFA. In 15 cases $(13.15 \%)$ MCFA arose from FA. Here we found 3 cases (5.25\%) on the right side and $2(3.5 \%)$ cases on the left side MCFA arising from FA at the same level as PFA, whereas 2 cases (3.5) on left side MCFA arose from FA superior to origin of PFA. 2 cases $(3.5$ $\%$ ) on the left side were found to arise from FA below the level of origin of PFA. We found 3 cases (5.25\%) in which MCFA arose from the FA as a common trunk with PFA. Figure 1 shows the varying origins of MCFA.

In case of LCFA, we observed that LCFA took origin from PFA in 48 cases ( $84 \%$ ) on the right side and in 40 cases $(70.18$ $\%$ ) on left side giving a mean of $77.09 \%$. In 6 cases (10.5\%) LCFA took origin from the FA at the same level as that of PFA on the right side and in 17 cases (29.82\%) on the left side. Out of the total number of cases, in 5 cases MCFA (4.38\%) and in 2 cases $(1.75 \%)$ LCFA was found to take origin superior to PFA. In 2 cases, we found MCFA to arise inferior to the origin of PFA whereas no such position was noted in LCFA. A common stump of origin was noted in 1 case $(1.75 \%)$ on the right side. 

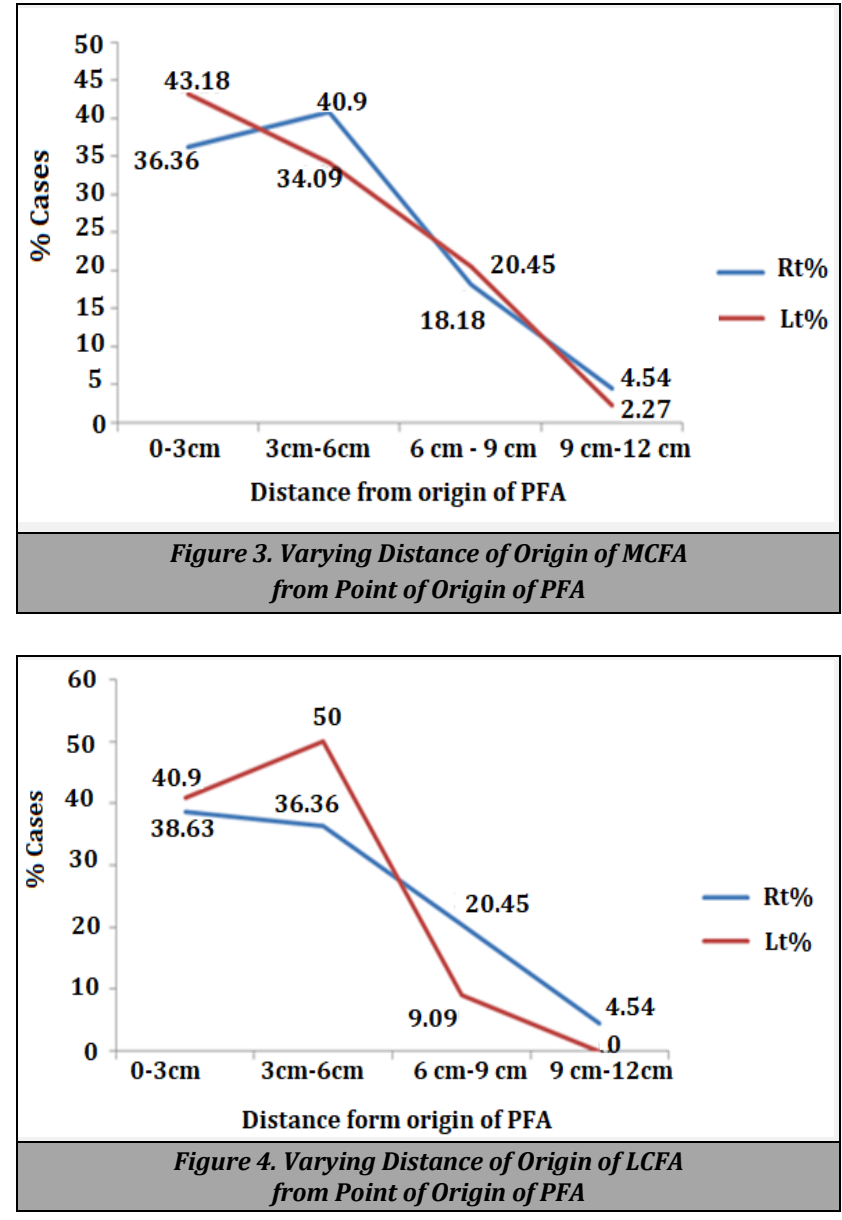

Figures $3 \& 4$ show the distance of origin of MCFA and LCFA from point of origin of PFA. The distance of origin of MCFA from point of origin of PFA was $3-6 \mathrm{~cm}$ in 18 cases ( $40.90 \%), 0-3 \mathrm{~cm}$ in 19 cases (43.18\%) in LCFA. We found a descending and transverse branch to arise as a common trunk from LCFA on right side, and a direct descending branch from femoral artery. Direct descending branch of LCFA from femoral artery was seen in one case. They have also reported a rare case of LCFA arising from external iliac artery. In a study done on the variation in the origin of PFA and its femoral triangles by Dixit et al. from dissecting 228 femoral triangles it was found that the MCFA originates at a higher level from PFA as compared to LCFA. They also found that the MCFA sometimes arises from FA and this incidence was higher than that of LCFA. Vuksanović-Božarićetal ${ }^{12}$ also studied the variability of the origin of circumflex femoral arteries. They performed microdissection of both lower limbs of 30 foetuses of varying gestational age from 7 - 10 lunar months. Their result showed that for most of the cases LCFA arise from the DFA. They reported that in $11.7 \%$ of cases MCFA had its origin from the CFA, in $5 \%$ cases from SFA and in one case the MCFA was missing.

\section{DISCUSSION}

It is crucial in surgical procedures to correctly make an incision to expose PFA and its branches. MCFA is the main artery that supplies the head and neck of the femur. While doing surgery for fractures or angiographic diagnostic procedures, in the region, the surgeon should be aware of the variations of MCFA in order to avoid iatrogenic injury to the vessel. The perforating branches of PFA together with the branches of MCFA and LCFA form an important collateral circulation which maintains circulation to the lower limb in case of obstruction of FA proximal to the origin of PFA. Thus, it is clinically very significant to know the site and pattern of origin of MCFA and LCFA from the PFA and their variations.

Normally MCFA and LCFA arise from PFA. In a cadaveric study in south Indian population it was found that on the right side, MCFA arose in $40 \%$ of cases from PFA and in $50 \%$ of cases from FA. However, on the left side in $60 \%$ of the cases MCFA arose from PFA and in $35 \%$ from FA. In the case of LCFA on the right side $80 \%$ of cases arose from PFA and $20 \%$ from FA and in left side $70 \%$ from PFA and $25 \%$ from FA. A lot of similar studies were also reported. ${ }^{1}$

In another study, $20 \%$ of MCFA arose from PFA and $80 \%$ from FA. ${ }^{21}$ In yet another report, $86 \%$ MCFA arose from PFA, while in another report it was $5 \% 22$ from FA. The incidence of origin of MCFA from FA was also reported from $5 \%$ to $80 \%$ or more in various studies. ${ }^{1}$ Rarer variations in the origin of MCFA have been reported like origin from superficial femoral artery, external iliac artery and from LCFA.

In a study of 221 embalmed cadavers, LCFA and MCFA was classified into three different patterns based on the origin. It was found that both arteries originated from PFA in $78.8 \%$ cases and in $20.5 \%$ one artery arose from PFA and the other from FA and in 0.5 cases both arteries arose from FA. ${ }^{23}$

100 computed tomography (CT) angiograms of the hip region were analysed and found that $50 \%$ of cases of MCFA and LCFA originated from PFA. In $31 \%$ MCFA arose from FA and LCFA from PFA. ${ }^{24}$ Thus, the study reported a lot of variations based on different race and region. In a review published in 2020, the authors assessed all the studies on PFA and its circumflex branches, after 2009, they found that MCFA and LCFA originated from PFA in $63.12 \%$ and $74.92 \%$ cases respectively. In $27 \%$ cases MCFA originated from CFA and LCFA originated from CFA in $12.12 \%$ cases. $^{25}$

In the present study we have observed several variations in the site and pattern of origin of MCFA and LCFA. The knowledge of the variants helps also to reduce intra-operative and post-operative complications.

\section{CONCLUSIONS}

Many authors have conducted studies on variability of circumflex femoral arteries. Their results have shown that LCFA and MCFA show wide variation in the source of origin and the distance of origin. Our study showed that circumflex branches of profunda femoris artery exhibits wide variations. In majority of cases, both MCFA and LCFA ( $89 \%$ of MCFA and $71 \%$ of LCFA) arose at a distance of $3-6 \mathrm{cms}$ from the origin of PFA from FA. In view of the aforementioned variations in the site and origin of MCFA and LCFA, it would be wise to define vascular pattern before performing any invasive procedure.

\section{Limitations}

In the occurrence of certain variations, we have not studied the gender differences in the origin of the PFA and its branches. In 
our future studies, we intend to look at the gender and racial differences in the origin of PFA and its branches.

Data sharing statement provided by the authors is available with the full text of this article at jemds.com.

Financial or other competing interests: None.

Disclosure forms provided by the authors are available with the full text of this article at jemds.com.

We acknowledge Dr. Shana Baby, Postgraduate student, and Dr. Anju George, Assistant Professor Department of Anatomy, Medical College Kottayam, Kerala, India, for the technical support.

\section{REFERENCES}

[1] Manjappa T, Prasanna LC. Anatomical variations of the profunda femoris artery and its branches-a cadaveric study in south Indian population. Indian J Surg 2014;76(4):288-92.

[2] Datta AK. Essentials of human anatomy: superior and inferior extremities. $5^{\text {th }}$ edn. Vol. 3. Current Books International 2009:145-6.

[3] Last RJ. Anatomy: regional and applied. $5^{\text {th }}$ edn. Published Edinburgh, London: Churchill Livingstone 1972.

[4] Lumsden AB, Colborn GL, Skandalakis LJ, et al. Surgical anatomy of the deep femoral artery. In: Surgery of the Deep Femoral Artery. Berlin, Heidelberg: Springer 1994:1-21.

[5] Standring S, Ellis H, Healy J, et al. Gray's anatomy: the anatomical basis of clinical practice. American Journal of Neuroradiology 2005;26(10):2703-4.

[6] Vazquez MT, Murillo J, Maranillo E, et al. Patterns of the circumflex femoral arteries revisited. Clin Anat 2007;20(2):180-5.

[7] Başkaya MK, Kiehn MW, Ahmed AS, et al. Alternative vascular graft for extracranial-intracranial bypass surgery: descending branch of the lateral circumflex femoral artery. Neurosurg Focus 2008;24(2):E8.

[8] Sugawara Y, Sato O, Miyata T, et al. Utilization of the lateral circumflex femoral artery as a midway outflow for aorto-popliteal grafting: report of a case. Surg Today 1998;28(9):967-70.

[9] Gradman WS. Bypass to the lateral circumflex femoral artery. Ann Vasc Surg 1992;6(4):344-6.

[10] Gautier E, Ganz K, Krügel N, et al. Anatomy of the medial femoral circumflex artery and its surgical implications. J Bone Joint Surg Br 2000;82(5):679-83.

[11] Bergman RA. Compendium of human anatomic variation: text, atlas and world literature. Baltimore: Urban \& Schwarzenberg 1988.
[12] Vuksanović-Božarić A, Abramović M, Vučković L, et al. Clinical significance of understanding lateral and medial circumflex femoral artery origin variability. Anat Sci Int 2018;93(4):449-55.

[13] Dixit D, Kubavat DM, Rathod SP, et al. A study of variations in the origin of profunda femoris artery and its circumflex branches. Int J Biol Med Res 2011;2(4):1084-9.

[14] Demirseren E, Gokrem S, Ozdemir OM, et al. Hatchet shaped tensor fascia lata musculocutaneous flap for the coverage of trochanteric pressure sores: a new modification. Ann Plast Surg 2003;51(4):419-22.

[15] Hollinshead HW. Textbook of Anatomy. $3^{\text {rd }}$ edn. Hagerstown, Maryland: Harper \& Row 1974: p. 407.

[16] Fernandes R, Lee J. Use of the lateral circumflex femoral artery perforator flap in the reconstruction of gunshot wounds to the face. J Oral Maxillofac Surg 2007;65(10):1990-7.

[17] Tanyeli E, Üzel M, Yildirim M, et al. An anatomical study of the origins of the medial circumflex femoral artery in the Turkish population. Folia Morphol (Warsz) 2006;65(3):209-12.

[18] Sabnis AS. Anatomical variations of profunda femoris artery. Journal of Clinical Research Letters 2013;4(1):546.

[19] Singh R. Variation of lateral circumflex femoral artery and its possible clinical and diagnostic implications. Int J Anat Var 2017;1(4):4-5.

[20] Savithri P. A rare variation of trifurcation of right femoral artery. Int J Anat Var 2013;6(1):4-6.

[21] Emura S, Shoumura S, Ishizaki N, et al. The anatomical study on the branches of the femoral artery (II). Comparison with the findings of Adachi's classification. Kaibogaku Zasshi 1989;64(3):196-205.

[22] Siddharth P, Smith NL, Mason RA, et al. Varia tional anatomy of the deep femoral artery. Anat Rec 1985;212(2):206-9.

[23] Massoud TF, Fletcher EW. Anatomical variants of the profunda femoris artery: an angiographic study. Surgical and Radiologic Anatomy 1997;19(2):99-103.

[24] Zlotorowicz M, Czubak-Wrzosek M, Wrzosek P, et al. The origin of the medial femoral circumflex artery, lateral femoral circumflex artery and obturator artery. Surg Radiol Anat 2018;40(5):515-20.

[25] Tzouma G, Kopanakis NA, Tsakotos G, et al. Anatomic variations of the deep femoral artery and its branches: clinical implications on anterolateral thigh harvesting. Cureus 2020;12(4):e7867. 